\title{
Nevanlinna theory, fuchsian functions and brownian motion windings
}

\section{Jean-Claude Gruet}

\begin{abstract}
Atsuji proposed some integrals along Brownian paths to study the Nevanlinna characteristic function $T(f, r)$ when $f$ is meromorphic in the unit disk $D$. We show that his criterion does not apply to the basic case when $f$ is a modular elliptic function. The divergence of similar integrals computed along the geodesic flow is also proved.
\end{abstract}

\section{Introduction.}

The Nevanlinna theory aims at giving a unified way to study the distribution properties of meromorphic functions in the plane $\mathbb{C}$ or in the unit disk $D$. We are concerned here with the disk case. We refer the reader to Nevanlinna's (1970) definitive monograph. If $D_{r}$ denotes the euclidean disk centered at 0 with radius $r<1$, Nevanlinna considered the following counting function

$$
N_{f}(r, a)=\sum_{\substack{f(z)=a \\ z \in D_{r}}} \log \left(\frac{r}{|z|}\right), \quad \text { the sum counting with multiplicity }
$$

2000 Mathematics Subject Classification: 60J65 30D35 30F45.

Keywords: Nevanlinna theory, meromorphic functions, Brownian motion, geodesic flow. 
Another fundamental quantity is the Nevanlinna characteristic (in the form of Ahlfors and Shimizu)

$$
T_{f}(r)=T(f, r)=\frac{1}{\pi} \int_{D_{r}} \frac{\left|f^{\prime}(z)\right|^{2}}{\left(|f(z)|^{2}+1\right)^{2}} \log \left(\frac{r}{|z|}\right) d x d y
$$

These two functions bear a probabilistic interpretation in terms of some complex Brownian motion (Carne, 1986). Atsuji (1995) extended Carne's proof to the case of harmonic maps between manifolds.

Define the deficiency index $0 \leq \delta(a) \leq 1$ for any value $a$ in the extended plane $\mathbb{C}_{\infty}$

$$
\delta(a)=\liminf _{r \rightarrow 1}\left(1-\frac{N_{f}(r, a)}{T_{f}(r)}\right)
$$

If $\delta(a)=1$ we say that $f$ omits the value $a$. This is a generalization of $f^{-1}(\{a\})=\varnothing$. A useful consequence of Nevanlinna's second theorem is the following:

Theorem (The Nevanlinna defect inequality). Let $f$ be a non constant meromorphic function on $D$ such that $T$ is unbounded and $a_{1}, \ldots, a_{q} q$ distinct values in $\mathbb{C}_{\infty}$. Then

$$
\sum_{i=1}^{q} \delta\left(a_{i}\right) \leq 2+\limsup _{r \rightarrow 1} \frac{\log \left(\frac{1}{1-r}\right)}{T_{f}(r)}
$$

A striking consequence is the Picard's type result for meromorphic functions: if $f$ omits $q$ values and

$$
\limsup _{r \rightarrow 1} \frac{\log \left(\frac{1}{1-r}\right)}{T_{f}(r)}<q-2,
$$

then $f$ is constant on $D$.

Hence some probabilistic interpretation of this limsup would be of great interest. Recall that the (upper) order of a meromorphic function

$$
\rho:=\limsup _{r \rightarrow 1} \frac{\log \left(T_{f}(r)\right)}{\log \left(\frac{1}{1-r}\right)}
$$


was characterized by means of killed Brownian motion by Carne (1986). We will be concerned mostly with the case $\rho=0$. Meromorphic functions with a characteristic function of a slow growth such that

$$
\limsup _{r \rightarrow 1} \frac{T_{f}(r)}{\log \left(\frac{1}{1-r}\right)}
$$

is finite were intensively studied by L. R. Sons (1984), D. F. Shea \& L. R. Sons (1986).

Atsuji (1996) proposed to use the parametrised family of integrals

$$
J_{\alpha}(f)=\int_{0}^{\sigma}\left|f\left(z_{s}\right)\right|^{\alpha} d s, \quad \alpha>0,
$$

if $\sigma$ the first hitting time of $\partial D$ by a complex Brownian $\left(z_{s}, s \geq 0\right)$ motion started in $D$. His motivation originated in the study of minimal surfaces.

We show in Section 2 that the size of $T_{f}$ does not seem to be easily related to the nature of the integrals $J_{\alpha}$. We consider in particular the example of the modular function which is strongly connected to our work on windings of the Brownian motion on the thrice punctured sphere (1998). Section 3 is devoted to analogous integrals when the geodesic flow serves as a substitute for the Brownian motion. We examine in section four how to recover some partial information from other integrals if $f$ belongs to the class of Fuchsian functions. We begin the paper by some results deduced from the probabilistic representation of Nevanlinna characteristic function. We take the opportunity to give a new result on normal meromorphic functions in the disk.

\section{Review on Nevanlinna theory and hyperbolic geo- metry.}

We replace sometimes Brownian motion in the disk by the hyperbolic Brownian motion in $D$ or in the Poincaré half-plane $\mathbb{H}$ which is better suited to our purpose. Hence we recall also known facts on hyperbolic geometry. 


\subsection{Some basic facts in Nevanlinna theory.}

We call spherical Brownian motion in $\mathbb{C}$ any planar Brownian motion run with a spherical change of time, i.e. any diffusion generated by

$$
\frac{1}{2} \Delta_{\mathrm{sph}}=\frac{1}{2}\left(1+|z|^{2}\right)^{2}\left(\frac{\partial^{2}}{\partial x^{2}}+\frac{\partial^{2}}{\partial y^{2}}\right)
$$

Let $f$ be a meromorphic function on $D$ and $\left(z_{s}, s \geq 0\right)$ a planar Brownian motion started at 0 . The generalized Lévy's theorem on time changes give us the existence of some spherical Brownian motion $\Sigma$ started at 0 such that

$$
f\left(z_{t}\right)=\Sigma\left(A_{t}\right) \text { with } A_{t}=\int_{0}^{t} \frac{\left|f^{\prime}\left(z_{s}\right)\right|^{2}}{\left(1+\left|f\left(z_{s}\right)\right|^{2}\right)^{2}} d s, \text { and } t<\sigma .
$$

Denote by $T_{r}$ the first hitting time by $z_{t}$ of the circle centered at 0 with radius $r<1$. Carne observed that $T_{f}(r)$ is simply $\mathbb{E}_{0}\left(A\left(T_{r}\right)\right)$.

As a matter of fact, the hyperbolic metric on $D$ is more natural than the euclidean one. Hence we consider a different set of notations when the disk $D$ is endowed with the hyperbolic metric

$$
\frac{4}{\left(1-|z|^{2}\right)^{2}}\left(d x^{2}+d y^{2}\right)
$$

Let $\left(\pi_{s}, s \geq 0\right)$ be some hyperbolic Brownian motion in $D$ started at 0 . We obtain the hyperbolic analogue of formula (1) for any time $t$

$$
f\left(\pi_{t}\right)=\Sigma\left(C_{t}\right) \text { with } C_{t}=\frac{1}{4} \int_{0}^{t} \frac{\left|f^{\prime}\left(\pi_{s}\right)\right|^{2}}{\left(1+\left|f\left(\pi_{s}\right)\right|^{2}\right)^{2}}\left(1-\left|\pi_{s}\right|^{2}\right)^{2} d s .
$$

Remark. If $T_{f}(1):=\lim _{r \rightarrow 1-} T_{f}(r)<\infty$, then $A\left(T_{1}\right)<\infty$ and $C_{\infty}<\infty$, almost surely. Hence $\left\{f\left(z_{s}\right), s \leq \sigma\right\}$ is almost surely a bounded set.

\subsection{The half plane model.}

Although the disk $D$ and the upper half plane $\mathbb{H}=\{\Im m(z)>0\}$ are two models of the same geometric object, we will use a capital letter $\Pi$ for the Brownian motion on $\mathbb{H}$ to distinguish it from the hyperbolic Brownian motion in $D$. We denote by $d(\cdot, \cdot)$ the hyperbolic distance in $D$ and $\mathbb{H}$. 
Let $I$ be the 1-1 conformal map between $\mathbb{H}$ and $D$ given by $I(z)=$ $(z-i) /(z+i)$. A hyperbolic Brownian motion on $\mathbb{H}$ is a diffusion $\left(\Pi_{u}, u \geq 0\right)$ with infinitesimal generator half of the hyperbolic Laplacian

$$
\mathcal{G}^{0}=\frac{1}{2} \Delta_{\mathbb{H}}=\frac{1}{2} y^{2}\left(\frac{\partial^{2}}{\partial x^{2}}+\frac{\partial^{2}}{\partial y^{2}}\right) .
$$

If $X$ and $Y$ denote the real and the imaginary part of $\Pi$, there exists two independent linear Brownian motions $B$ and $\beta$ started at 0 such that

$$
Y_{u}=Y_{0} \exp \left(B_{u}-\frac{u}{2}\right) \text { and } X_{u}=X_{0}+\beta\left(\int_{0}^{u} Y_{0}^{2} \exp \left(2 B_{s}-s\right) d s\right) .
$$

\subsection{The modular function $\lambda$.}

The modular function $\lambda$, also denoted $k^{2}$ (Ahlfors, Section 3.4 (1966) or Ford (1951)) is a $\Gamma_{2}$ periodic analytic function on $\mathbb{H}$. $\Gamma_{2}$ is the group of hyperbolic isometries

$$
z \longrightarrow \frac{a z+b}{c z+d}
$$

such that $a, b, c$ and $d$ are integers with $a d-b c=1$ and

$$
\left(\begin{array}{ll}
a & b \\
c & d
\end{array}\right) \equiv\left(\begin{array}{ll}
1 & 0 \\
0 & 1
\end{array}\right) \quad \bmod 2
$$

$\Gamma_{2}$ which is called the modular group of second level is a free nonabelian group generated by the transforms $T: z \longrightarrow z+2$ and $S: z \longrightarrow z /(2 z+1)$.

Let the domain $\Delta$ be $\{z: 0<\Re e(z)<1,|1 / 2-z|>1 / 2\}$ and let $\Delta^{\prime}$ be its reflection by $(O y)$. The set $\mathcal{F}_{0} \stackrel{\text { (def) }}{=} \Delta^{\prime} \cup \bar{\Delta}$ is a $\Gamma_{2}$ fundamental region because any point in $\mathbb{H}$ belongs to a unique translate $g \mathcal{F}_{0}\left(g \in \Gamma_{2}\right)$.

$\lambda$ is a 1-1 conformal mapping of the hyperbolic triangle $\Delta$ on the upper half plane in $\mathbb{C}$. $\lambda$ is unique if we require $\lambda(0)=1, \lambda(1)=\infty$ and $\lambda(\infty)=0$. We know from Ahlfors, p. 272, that

$$
\begin{aligned}
& |\lambda(z)| \sim 16 \exp (-\pi \Im m(z)) \\
& \text { uniformly when } \Im m(z) \text { goes to infinity . }
\end{aligned}
$$

From now on, we consider instead of $\lambda$ the elliptic function $\mu=1-1 / \lambda$ such that $\mu(z)=\lambda(1-1 / z)$ (Ahlfors formula (25)). This function enjoys the same properties except that the vertices of the triangle $\Delta$ are exchanged. 
Notation. The function $h:=\mu \circ I^{-1}$ is an automorphic function with respect to the group $G_{0}$ acting on $D$, conjugated to $\Gamma_{2}$.

The asymptotic of $T\left(\mu \circ I^{-1}, r\right)$ is well known:

Theorem (Tsuji, XI.29). T( $\left.\mu \circ I^{-1}, r\right) \sim \log (1 /(1-r))$ when $r$ increases to 1 . Hence the Nevanlinna defect inequality is sharp because $\mu$ omits only the three values $\infty, 0$ and 1 .

We provide two proofs of this basic result. The first one which relies on orbits counting is classical; but unlike Tsuji's (1959) approach via potential theory, orbits counting is directly applied. The second proof is a variant of Tsuji's method. Instead of the geodesic flow, we use a Brownian motion. We get an almost sure limit and therefore we can conclude like Tsuji's Theorem XI.28 that the modular function enjoys an almost radial behavior.

First proof. The argument is based on a non euclidean Weyl's type estimate (Patterson, 1975) obtained with the help of a trace formula: for any $z$ in $D$, when $r$ goes to one, $\#\left\{g \in G_{0} / g(z) \in D_{r}\right\} \sim 1 /(1-r)$ or $\exp (d)$ in terms of the hyperbolic radius $d$ of $D_{r}, d=\log ((1+r) /(1-r))$, equivalent to $\log (1 /(1-r))$.

Consider the ordinary counting function associated to the value $a$ in the target space $\mathbb{C}_{\infty}=\mathbb{C} \cup\{\infty\}: n_{f}(r, a)=\#\left\{z \in D_{r}, f(z)=a\right\}$, the sum counting with multiplicity. By means of an integration by parts, we obtain $N_{f}(r, a) \sim \int_{0}^{r} n_{f}(u, a) d u$ provided that $n_{f}(\cdot, a)$ goes to infinity. Choose any $a$ different from 0,1 and $\infty$. Then $N_{\mu \circ I^{-1}}(r, a)$ is equivalent to $\log (1 /(1-r))$. Recall now the classical theorem (Tsuji V.5 for instance): if $f$ is meromorphic on $D$ with an unbounded Nevanlinna characteristic, $T_{f}(r)$ is equivalent to $N_{f}(r, a)$ when $r$ goes to 1 , except a set of capacity zero of exceptional values $a$ in the sphere $\mathbb{C}_{\infty}$.

Second Proof. In fact a much stronger convergence holds:

Lemma 1.1. Let $f$ be the function $\mu \circ I^{-1}$. In this particular case, we have

$$
\lim _{t \rightarrow \infty} \frac{C_{t}}{t} \stackrel{\text { a.s. }}{=} \frac{1}{2} \text {. }
$$

Hence $\mathbb{E}\left(C\left(\widehat{\sigma}_{d}\right)\right) \sim d$ where $\widehat{\sigma}_{d}=\inf \left\{s \geq 0: d\left(\pi_{0}, \pi_{s}\right)=d\right\}$. Thus $T_{f}(r)=\mathbb{E}\left(A\left(T_{r}\right)\right) \sim \log (1 /(1-r))$ when $r$ goes to 1 . Moreover

$$
\frac{A\left(T_{r}\right)}{\log \left(\frac{1}{1-r}\right)} \stackrel{\text { a.s. }}{\longrightarrow} 1 \text {. }
$$


Proof. By an easy computation the function

$$
z \longrightarrow\left(1-|z|^{2}\right) \frac{\left|f^{\prime}(z)\right|}{1+|f(z)|^{2}}
$$

is $G_{0}$ invariant. Hence $\left(C_{t}\right)$ is an integrable additive functional of the recurrent hyperbolic Brownian motion $\left(\pi_{s}^{\prime}, s \geq 0\right)$ on $D / G_{0}$. But this Brownian motion is ergodic with respect to the Haar measure on $I\left(\mathcal{F}_{0}\right)$ and the above functional is integrable since

$$
\int_{I\left(\mathcal{F}_{0}\right)} \frac{\left|f^{\prime}(z)\right|^{2}}{\left(1+|f(z)|^{2}\right)^{2}} d x d y=\int_{\mathbb{C}} \frac{d x d y}{\left(1+|z|^{2}\right)^{2}}=\pi .
$$

Hence $C_{t} / t$ almost surely goes to $\pi / \operatorname{area}\left(\mathcal{F}_{0}\right)=1 / 2$.

This computation already appeared in Proposition 2.2, Gruet (1998), but we used a different definition of the spherical Brownian motion.

Now

$$
\mathbb{E}\left(C_{t}\right) \sim \frac{1}{2} t
$$

by $L^{1}$ convergence. But

$$
2 \frac{d\left(\pi_{t}, \pi_{0}\right)}{t} \stackrel{\text { a.s. }}{\longrightarrow} 1
$$

hence for any $\varepsilon$, if $d$ is great enough $(2-\varepsilon) d \leq \widehat{\sigma}_{d} \leq(2+\varepsilon) d$. Since $A\left(T_{r}\right)=C\left(\widehat{\sigma}_{d}\right)$ by time change, we obtain $T_{f}(r) \sim d$.

\subsection{Fuchsian functions.}

We consider automorphic functions on $X=D$ or $\mathbb{H}$ associated to finitely generated Fuchsian groups $G$ of first kind. We denote by $\mathcal{F}$ a fundamental finite sided polygon: the vertices belonging to $\partial X$ are parabolic whereas the others are called elliptic. We suppose that $f$ tends to a limit in $\mathbb{C} \cup \infty$ at each parabolic vertex $p_{i}$. This implies meromorphy at $p_{i}$ (see Lehner (1964) for instance). According to the old terminology of L. R. Ford (1951), these functions are simple.

By the very definition of meromorphy at a parabolic vertex, if $\infty$ is a parabolic vertex of a automorphic function $g$ defined on $\mathbb{H}$ such that

$$
g(\infty)=\infty, \quad|g(z)| \sim a_{0} \exp \left(\frac{2 \pi n}{c} \Im m(z)\right)
$$


when $\Im m(z)$ goes to infinity. $a_{0}$ is some positive constant, $c$ denotes the circumference of the cusp (Ratcliffe p. 423) and $n$ is the valence of $g$ : this means that $g$ assumes each value $n$ times on $\mathcal{F}$. For instance, when $g=\mu$, $c=2$ and $n=1$.

If $f$ is defined on $D$, the circumference of a cusp at a parabolic vertex $p$ is defined after passing to the upper half plane model and sending $I^{-1}(p)$ to $\infty$. We denote by $H(p, R)$ the horoball in $D$ conjugated to $I(]-c, c] \times$ $[R, \infty[)$.

We extend easily our first proof of Tsuji's theorem XI.29 to the general case with the help of Patterson's result

$$
\#\left\{g \in G / g(z) \in D_{r}\right\} \sim \frac{2 \pi}{\operatorname{area}(\mathcal{F})} \frac{1}{1-r} .
$$

Hence if the fundamental polygon of $f$ has finite area,

$$
T_{f}(r) \sim \frac{2 n \pi}{\operatorname{area}(\mathcal{F})} \log \left(\frac{1}{1-r}\right) .
$$

\subsection{Normal meromorphic functions.}

Recall that a meromorphic function $f$ defined in $D$ is normal if the family $\left(f \circ T, T \in G_{0}\right.$ ) is normal, i.e. relatively compact with respect to the uniform convergence on compact sets on $D$. The target space $\mathbb{C}_{\infty}$ is endowed with the chordal distance.

According to Noshiro's theorem (e.g. Schiff's (1993) monograph), $f$ is normal if and only if

$$
\left(1-|z|^{2}\right) \frac{\left|f^{\prime}(z)\right|}{|f(z)|^{2}+1}
$$

is bounded in $D$. Obviously simple automorphic functions of first kind are normal (some extensions are discussed in Pommerenke (1974)).

Theorem. Let $f$ be a normal meromorphic function in $D$ and $\phi$ an increasing positive function such that $\int^{\infty} d t / \phi(t)$ is convergent. Then for almost any $\theta$,

$$
\lim _{r \rightarrow 1} \frac{\log \left(\left|f\left(r \exp ^{i \theta}\right)\right|\right)}{\phi\left(\log \left(\frac{1}{1-r}\right)\right)}
$$

vanishes. 
Proof. This is only a slight modification of Lyons' proof (1990) of Makarov's law of the iterated logarithm. Denote by

$$
C(f):=\sup _{z \in D}\left(1-|z|^{2}\right) \frac{\left|f^{\prime}(z)\right|}{|f(z)|^{2}+1}<\infty \text {. }
$$

Recall the representation $f\left(\pi_{t}\right)=\Sigma\left(C_{t}\right)$ with $C_{t} \leq C(f)^{2} t$ for all $t$. Let us identify $\left(\Sigma_{s}, s \geq 0\right)$ and its stereographic projection on the extended plane. The radial part is a diffusion with scale function $S(r)=\log (r)$ and speed measure

$$
m(r) d r=\frac{r}{\left(1+r^{2}\right)^{2}} d r
$$

We first show the almost sure estimate

$$
\limsup _{s \rightarrow \infty} \frac{\log \left(\left|\Sigma_{s}\right|\right)}{\phi(s)}=0, \quad \text { if } \int^{\infty} \frac{d t}{\phi(t)}<\infty .
$$

Indeed, by Motoo's lemma (see e.g. Itô and McKean book), if $h$ is an increasing positive function such that

$$
\int^{\infty} \frac{1}{\log (h(s))} d s<\infty
$$

then, almost surely, $\left|\Sigma_{t}\right|<h(t)$ if $t$ is great enough. Note that Motoo's argument remains true although the scale function $S$ goes to infinity at 0 and $+\infty$ because the spherical Brownian motion is positive recurrent.

Thus

$$
\limsup _{t \rightarrow \infty} \frac{\log \left(\left|f\left(\pi_{t}\right)\right|\right)}{\phi(t)} \leq 0
$$

since the integral test is convergent for any $\alpha \phi$ where $\alpha$ is arbitrary small. Furthermore, the above ratio goes to zero since $1 / f$ is also a normal function. Note also that

$$
\phi(t) \leq \phi\left(\log \left(\frac{1}{1-\left|\pi_{t}\right|}\right)\right)
$$

when $t$ goes to infinity. To finish the proof we use the fact that if a function on the disk goes to zero along almost any Brownian path (fine limit), then the function has the same limit 0 along almost any ray. 


\section{Remarks:}

a) Let $f$ be analytic and normal. Usually the quantity

$$
M(r, f)=\sup _{z \in D_{r}}|f(z)|
$$

is much bigger, roughly of order $\exp ($ Const $/(1-r))$. A more precise bound is given by Schiff's Theorem 3.6.10. The optimal bound for the modular function comes from the equivalent $(*)$ of Section 1.3.

b) If something like

$$
\liminf _{t \rightarrow \infty} \frac{C_{t}}{t}>\text { const }>0
$$

is known, we could use the divergent case in Motoo's computation. Hence by Lemma 1.1, the integral test is optimal when $f$ is the modular function. We obtain for these kind of functions the divergent counterpart: if $\int^{\infty} d t / \phi(t)=\infty$, then for almost any $\theta$,

$$
\limsup _{r \rightarrow 1} \frac{\log \left(\left|f\left(r \exp ^{i \theta}\right)\right|\right)}{\phi\left(\log \left(\frac{1}{1-r}\right)\right)}>0
$$

\section{The counter-examples.}

\subsection{The modular function case.}

Theorem 2.1. All the integrals $J_{\alpha}\left(\mu \circ I^{-1}\right)$ are almost surely infinite although

$$
\limsup _{r \rightarrow 1} \frac{\log \left(\frac{1}{1-r}\right)}{T_{\mu \circ I^{-1}}(r)} \quad \text { is equal to one. }
$$

A key observation is that $J_{\alpha}\left(\mu \circ I^{-1}\right)$ is in fact a functional of the quotient Brownian motion $\pi^{\prime}$ on $D / G_{0}$. According to a well-known method (Lyons-McKean, Franchi, Gruet) we decompose the trajectory into excursions.

The first step is to use the Poincaré model.

Lemma 2.2 (Atsuji, 1996). Let $\left(z_{t}, t \geq 0\right)$ be a complex Brownian motion started at $z_{0}$ in $D$. Denote by $\sigma$ the first hitting time of $\partial D$. Then 
for any hyperbolic Brownian motion on the Poincaré half plane, started at $I^{-1}\left(z_{0}\right)$, the integrals $J_{\alpha}(f)$ and $\int_{0}^{+\infty}\left|f \circ I\left(\Pi_{u}\right)\right|^{\alpha} Y_{u}^{2} d u$ converge or diverge together.

Proof. By Paul Lévy's theorem on conformal maps, $\left(I^{-1}\left(z_{t}\right), t \geq 0\right)$ is the time change of some hyperbolic Brownian motion $\left(\Pi_{s}, s \geq 0\right)$ on the Poincaré half plane $\mathbb{H}$. Thus $\left(I\left(\Pi_{s}\right), s \geq 0\right)=\left(z\left(Q_{s}\right), s \geq 0\right)$ where $\left(Q_{s}, s \geq 0\right)$ is the additive functional

$$
Q_{s}=\int_{0}^{s} \frac{4}{\left|\Pi_{u}+i\right|^{4}} Y_{u}^{2} d u
$$

Hence $\int_{0}^{\sigma}\left|f\left(z_{s}\right)\right|^{\alpha} d s$ becomes

$$
4 \int_{0}^{+\infty}\left|f \circ I\left(\Pi_{u}\right)\right|^{\alpha} \frac{Y_{u}^{2}}{\left|\Pi_{u}+i\right|^{4}} d u .
$$

Since $\Pi$ has almost surely a finite limit on the $(O x)$ axis when $u$ increases to infinity, we obtain that $J_{\alpha}$ has the same nature as

$$
\int_{0}^{+\infty}\left|f \circ I\left(\Pi_{u}\right)\right|^{\alpha} Y_{u}^{2} d u
$$

Remark. If $\int_{0}^{+\infty}\left|f \circ I\left(\Pi_{u}\right)\right|^{\alpha} e^{-2 u} d u$ is infinite, then $J_{\alpha}(f)=\infty$ because $Y_{u} \geq \exp (-u)$ when $u$ is great enough.

Hence if we denote by $\Pi_{s}^{\prime}$ the unique $\Gamma_{2}$ translate of $\Pi_{s}$ belonging to $\mathcal{F}_{0}$, we are led to study the integrals

$$
\int_{0}^{+\infty} \exp \left(\pi \alpha \Im m\left(\Pi_{s}^{\prime}\right)\right) \exp (-k s) d s
$$

where $k$ is an arbitrary positive constant.

Our theorem 2.1 is deduced from the estimate $(*)$ and from the following

Proposition 2.3. For any pair of positive $c$ and $k$, almost surely

$$
\int_{0}^{+\infty} \exp \left(c \Im m\left(\Pi_{s}^{\prime}\right)\right) \exp (-k s) d s=\infty .
$$


Proof. We decompose the trajectory of the Markovian process $\Pi^{\prime}$ into the successive downward crossings between the two levels $y=R$ and $y=R-\varepsilon$ with $0<\varepsilon<R$.

Denote by $\mathcal{L}_{2}$ the segment $\{y=R\} \cap \mathcal{F}_{0}$ and $\mathcal{L}_{1}$ the segment $\{y=$ $R-\varepsilon\} \cap \mathcal{F}_{0}$ and let us define inductively a sequence of stopping times

$$
\begin{gathered}
S_{1}=\inf \left\{t: \Pi_{t}^{\prime} \in \mathcal{L}_{2}\right\}, \\
T_{n}=\inf \left\{t>S_{n}: \Pi_{t}^{\prime} \in \mathcal{L}_{1}\right\}, \\
S_{n}=\inf \left\{t>T_{n-1}: \Pi_{t}^{\prime} \in \mathcal{L}_{2}\right\} .
\end{gathered}
$$

Since these segments are positive recurrent for the ergodic diffusion $\Pi^{\prime}$, the number of outward crossings before time $t$ is roughly proportional to the time elapsed. More precisely, if the constant $k_{1}$ is great enough, $S_{n} \leq k_{1} n$ when $n$ is greater than some random integer $N_{0}$.

By $\Gamma_{2}$ invariance, we can lift the "excursion" $\left(\Pi_{t}^{\prime}, S_{n} \leq t \leq T_{n}\right)$ with the help of some hyperbolic Brownian motion $\Pi^{(n)}$ started at $\Pi^{\prime}\left(S_{n}\right)$ in $\mathcal{F}_{0}$ and stopped on the infinite line $y=1$ in $\mathbb{H}$. Consider some hyperbolic Brownian motion $\Pi$ started at $R i$ and $T$ its first hitting time of the line $y=R-\varepsilon$. Denote by $\left(U_{n}, n \geq 1\right)$ the independent copies of $\int_{0}^{T} \exp \left(\pi \alpha \Im m\left(\Pi_{s}\right)\right) d s$ created by these excursions. The independence arises from the $(0 x)$ translational invariance. Hence

$$
\int_{0}^{+\infty} \exp \left(\pi \alpha \Im m\left(\Pi_{s}^{\prime}\right)\right) \exp (-k s) d s
$$

is underestimated by $\sum_{n \geq N_{0}} \exp \left(-k k_{1} \cdot n\right) U_{n}$.

We use the second Borel-Cantelli lemma to investigate this sum. Our Lemma 2.4 below implies that $\mathbb{E}\left(\log \left(U_{1}\right)\right)=+\infty$ and $\sum_{n} \mathbb{P}\left(U_{n}>L e^{k k_{1} n}\right)$ $=\infty$ for any positive constant $L$. Hence almost surely

$$
\sum_{n} \exp \left(-k k_{1} \cdot n\right) U_{n}=\infty
$$

and

$$
\sum_{n \geq N_{0}} \exp \left(-k k_{1} \cdot n\right) U_{n}=\infty
$$

Thus

$$
\int_{0}^{+\infty}\left|g\left(\Pi_{s}\right)\right|^{\alpha} \exp (-2 s) d s=\infty
$$

is proven. 
Lemma 2.4. Let $\left(b_{s}, s \geq 0\right)$ be a linear Brownian motion with drift $-1 / 2$ started at $v, c$ any positive number and

$$
I_{c} \stackrel{(\text { def })}{=} \int_{0}^{\sigma(u)} \exp \left(c \exp \left(b_{s}\right)\right) d s
$$

where $\sigma(u)=\inf \left\{s \geq 0, b_{s}=u\right\}$ is the first hitting time of the level $u<v$. Then for any $c>0$ we have $\mathbb{E}\left(\log \left(I_{c}\right)\right)=+\infty$.

Proof. By translational $(O y)$ invariance, we may suppose $u=-1$ and $v=0$ to simplify the notations. We show that there exists some positive constant $K$ such that for any large $x, \mathbb{P}\left(\log \left(I_{c}\right)>x\right) \geq K / x$ for some positive constant $K$.

Set $a=\log (x / c)$ and $b=a+1$. Since

$$
I_{c} \geq \mathbf{1}_{\{\sigma(b) \leq \sigma(-1)\}} \exp \left(c e^{a}\right)\left(\sigma(a) \circ \theta_{\sigma(b)}-\sigma(b)\right),
$$

we deduce from the Markov property that $\mathbb{P}\left(\log \left(I_{c}\right)>x\right)$ is underestimated by

$$
\mathbb{P}_{0}(\sigma(b) \leq \sigma(-1)) \mathbb{P}_{b}\left(\sigma(a) \geq \exp (x) \exp \left(-c e^{a}\right)\right) .
$$

But $\mathbb{P}_{b}(\sigma(a) \leq 1)$ is independent from $x$ and

$$
\mathbb{P}_{0}(\sigma(b) \leq \sigma(-1))=\frac{1-\exp (-1)}{\exp (b)-\exp (-1)} \sim \frac{\text { Const }}{x}
$$

Remark. The line above gives some explanation about the key role of Cauchy tails.

We obtain now a refinement to the "Sullivan's estimate" for the recurrent Brownian motion $\pi^{\prime}$ on $D / G_{0}$ :

Theorem (Sullivan, 1982). Let $d(\cdot, \cdot)$ be the hyperbolic distance, then

$$
\limsup _{t \rightarrow \infty} \frac{d\left(i, \pi_{t}^{\prime}\right)}{\log (t)}=1, \quad \text { almost surely }
$$

Theorem 2.5. For any pair of positive $k, c$, we have almost surely

$$
\int_{0}^{+\infty} \exp \left(c e^{d\left(\pi_{s}^{\prime}, i\right)}\right) \exp (-k s) d s=\infty \text {. }
$$


Proof. Since the hyperbolic distance $d\left(z, z^{\prime}\right)$ is defined by the formula (see Terras for instance)

$$
2 \cosh \left(d\left(z, z^{\prime}\right)\right)=\frac{\left(x-x^{\prime}\right)^{2}+y^{2}+y^{\prime 2}}{y y^{\prime}}
$$

we have $\exp \left(c d\left(\pi_{s}^{\prime}, i\right)\right) \sim \exp \left(c \Im m\left(\pi_{s}^{\prime}\right)\right)$ when $\Im m\left(\pi_{s}^{\prime}\right)$ goes to infinity.

Note that Sullivan actually stated the much more difficult result for the geodesic flow. Hence, we will examine the flow in the next Section 3.

\subsection{Brownian windings on the sphere.}

A better understanding of the preceding paragraph is gained if we recall that $(\mathbb{H}, \mu)$ is the universal covering of the plane minus two points or equivalently of the sphere $\mathbb{C}_{\infty}$ minus three points, say $p_{1}, p_{2}, p_{3}$. Since we only use a single cusp in $\mathcal{F}_{0}$, the McKean and Sullivan (1984) construction is simplified here. They defined the outward crossings between very small circles around $p_{i}$ and a larger one around $p_{i}$. These pieces of trajectory encapture most of the windings around the points $p_{i}$.

Equip the sphere $S^{2}$ with a spherical Brownian motion $\left(\Sigma_{t}, t \geq 0\right)$. Let $J$ be the classical inversion such that $J$ defines a one to one mapping $S^{2} \longrightarrow \mathbb{C}_{\infty}$. Although the $\mu \circ J^{-1}$ lift on $\mathbb{H}$ of $\Sigma$ is not a hyperbolic Brownian motion, these two processes are not very different. I have shown (Gruet, 1998) that they differ by an ergodic change of time.

We can consider our excursions from two points of view: they are the parts of the trajectory where $\pi$ lies in the vicinity of the set $\bigcup_{g \in G_{0}} g(\infty)$ but they are also the parts where $\Sigma$ dramatically winds around $J^{-1}(\infty)$.

Note that the variation of the winding number is connected to the horizontal component whereas our copies of $\int_{0}^{T} \exp \left(\pi \alpha \Im m\left(\Pi_{s}\right)\right) d s$ are related only to the vertical part.

\subsection{An example of order $k-1$.}

Consider the functions

$$
f_{k}(z)=\exp \left( \pm \frac{1}{(1-z)^{k}}\right)
$$


when the integer $k$ is greater than 2. We know from Tsuji p. 205 that

$$
\limsup _{r \rightarrow 1} \frac{\log \left(T\left(f_{k}, r\right)\right)}{\log \left(\frac{1}{1-r}\right)}=k-1
$$

this property means that the order of $f_{k}$ is $k-1$.

Proposition 2.6. $J_{\alpha}\left(f_{k}\right)$ remains finite for every positive $\alpha$; although $T\left(f_{k}, r\right)$ is much bigger than $T\left(\mu \circ I^{-1}, r\right)$.

Proof. When $u \longrightarrow+\infty,\left(\Pi_{u}\right)$ almost surely goes to a limit $\Pi_{\infty}$ distributed as a Cauchy law on the axis. Hence $\int_{0}^{+\infty}\left|f_{k} \circ I\left(\Pi_{u}\right)\right|^{\alpha} Y_{u}^{2} d u$ is, almost surely, convergent.

Hence we observe that the integrals $J_{\alpha}(f)$ provide very few information information about $T_{f}$, even if $f$ is analytic. They do not take into account the almost invariance by homographic transformations:

Fact (Tsuji's Theorem V.2, for instance). If $f$ is meromorphic on D, for any complex coefficients such that $a d-b c \neq 0$,

$$
T_{(a f+b) /(c f+d)}=T_{f}+O(1) .
$$

Moreover, we have even $T_{1 / f}=T_{f}$.

\section{The geodesic flow analogue.}

First recall the

Theorem (Logarithmic law for geodesics, Sullivan). For almost all starting directions $v$ of geodesics

$$
\limsup _{t \rightarrow \infty} \frac{v(t)}{\log (t)}=1
$$

where $v(t)$ denotes the distance in $\mathcal{F}_{0} / G_{0}$ from a fixed point to the point achieved after traveling time $t$ along the geodesic starting in direction $v$.

For recent improvements, see Melián and Pestana (1993) or Stratman and Velani (1995). 
We obtain now a improvement of the logarithmic law in the spirit of our theorem 2.5. As usual, the hyperbolic planar Brownian motion and the geodesic flow enjoy very similar behavior.

Theorem 3.1. Let $\left(\gamma_{s}, s \geq 0\right)$ be the geodesic flow on $\mathbb{H}$ started at $\gamma_{0}=i$ for instance. Denote by $\left(\gamma_{s}^{\prime}, s \geq 0\right)$ the projection on $\mathbb{H} / \Gamma_{2}$. Then for almost all starting directions, for any pair of positive $k, c$,

$$
\int_{0}^{+\infty} \exp \left(c e^{d\left(\gamma_{s}^{\prime}, i\right)}\right) \exp (-k s) d s=\infty
$$

Proof. We consider the excursions of the geodesic flow $\left(\gamma_{s}^{\prime}, s \geq 0\right)$ between the lines $y=2$ and $y=1$ in the set $D_{\infty}=\{\Im m(z) \geq 1,0 \leq \Re e(z) \leq 1\}$, namely the trace in $\mathcal{F}_{0}$ of the horoball $\{\Im m(z) \geq 1\}$.

Let $d_{n}$ be the maximum of $d\left(\gamma_{s}^{\prime}, i\right)$ during the $n$-th excursion in the set $\cup_{g \in G_{0}} g\left(D_{\infty}\right)$. Hence, if we draw the picture in the $D_{\infty}$ representative, the maximum penetration $d_{n}$ is attained at a point where the speed is horizontal. In analogy with the Brownian case, denote by $S_{n}$ and $T_{n}$ the first or last time of this excursion.

Consider the geodesic parameterized by the arc length $s$, started with horizontal speed at some point with hyperbolic distance $d$ from the line $y=1$

$$
\left(H \tanh (s), \frac{H}{\cosh (s)}\right), \quad \text { where } H=\exp (d)
$$

Hence the contribution of any excursion with $d=\log (H)$ penetration is at least

$$
\int_{0}^{\operatorname{argcosh}(H)} \exp \left(\frac{c H}{\cosh (s)}\right) d s=\int_{1}^{H} \exp \left(\frac{c H}{u}\right) \frac{d u}{\sqrt{u^{2}-1}}
$$

underestimated by

$$
\int_{1 / 2}^{1} \exp (c H v) \frac{d v}{v \sqrt{1-v^{2}}}
$$

when $H$ is large enough. The Laplace method says that this last integral is equivalent to $\exp (c H) \sqrt{\pi /(c H)}$. Thus the contribution of the $n$th excursion is underestimated by

$$
\text { Const } \frac{\exp \left(c \exp \left(d_{n}\right)\right)}{\sqrt{\exp \left(d_{n}\right)}} \exp \left(-k T_{n}\right) \text {. }
$$


Recall that the ergodic theorem implies that $T_{n}$ is bounded by some multiple of $n$ when $n$ goes to infinity. But the next lemma, deduced from Sullivan's work, does the job: if we apply it with $f(t)=\log (t)+\log (\log (t))$, we obtain that for almost any initial direction, there is infinitely many excursions in $D_{\infty}$ such that $\exp \left(d_{n}\right) \geq$ const $n \log (n)$.

Lemma 3.2 (deduced from Sullivan, 1982). Let $f$ be a lipschitzian positive increasing function defined on $\left[1, \infty\left[\right.\right.$. We have $d\left(\gamma_{s}, i\right) \geq f(s)$ infinitely often when $s$ increases to infinity if and only if $\int_{1}^{\infty} \exp (-f(t)) d t$ is divergent.

Proof. Sullivan's integral test is written in terms of the euclidean radius $x$ of the successive horoballs $h\left(D_{\infty}\right)$ (with $h$ in $G_{0}$ ) containing the points where maximum penetration is obtained. If the horoball is not an euclidean ball (viz $h$ is not a translation), $x$ is not defined, but this does not happen when the time goes to infinity.

Let $a$ be a function of $x \geq 1$ which is up to a bounded radio only depends of $\log (x)$. Then Sullivan proved that we have infinitely often penetrations at least $\log (1 / a(1 / x))$ if and only if $\int^{\infty}(a(x) / x) d x$ is divergent.

We have now to link the radius $x$ with the time $s$. There exists a constant $K>1$ such that $\exp (-(1 / K) s) \leq x \leq \exp (-K s)$ when $s$ goes to infinity.

Basically we have $f(t)=\log (1 /(a \exp (t)))$ and the integral test becomes $\int^{+\infty} \exp (-f(t)) d t=\infty$. Indeed by monotonicity, we can compare the integrals with discrete sums.

Remark. The Brownian analogue of this lemma is easily obtained with the help of the second Borel-Cantelli lemma and the estimate

$\mathbb{P}\left(\Im m\left(\pi^{\prime}\right)\right.$ reaches a level over $b$ during a given excursion $) \sim \exp (-b)$.

We get the following statement: let $f$ be an increasing positive function. $\Im m\left(\pi_{s}^{\prime}\right) \geq f(s)$ infinitely often when $s$ increases to infinity if and only if $\int_{1}^{\infty} \exp (-f(t)) d t$ is divergent.

\section{The integral criteria.}

Our task is to find some integral adapted to the behavior of Fuchsians functions near the parabolic points. Since we have met sums of i.i.d. random variables with "Cauchy-like" tails, it should be clear that in order to recover a constant in some limit result, we must use the well-known fact: 
Fact. Let $\left(X_{k}, k \geq 0\right)$ be i.i.d. positive random variables and $S_{n}=$ $\sum_{k=1}^{n} X_{k}$. Suppose that the tail $\mathbb{P}\left(X_{1} \geq x\right) \sim$ const $/ x$ when $x$ goes to infinity. Then $S_{n} /(n \log (n)) \stackrel{P}{\longrightarrow}$ const where $\stackrel{P}{\longrightarrow}$ denotes the convergence in probability.

Theorem 4.1. Let $f$ be a simple Fuchsian function of first kind on $D$ and valence $n$. Denote by $\mathcal{P}$ the set of parabolic points belonging to the fundamental region. Suppose that $f^{-1}(\infty) \cap \mathcal{P}$ contains $r$ points $p_{i}$ associated to the circumferences $c_{i}$, with the notations of Section 1.4. If

$$
I(f, t):=\int_{0}^{t} \log ^{+}\left(\left|f\left(\pi_{s}\right)\right|\right) d s
$$

we obtain that $I(f, t) /(t \log (t))$ converges in probability towards

$$
2 n \sum_{i=1}^{r} \frac{1}{c_{i}} .
$$

If $f^{-1}(\infty) \cap \mathcal{P}$ is empty, the limit above is zero.

Corollary. Let $f$ be a simple Fuchsian function on $D$ such that

$$
\frac{1}{t \log (t)} I\left(a f+\frac{b}{c f}+d, t\right) \stackrel{P}{\longrightarrow} 0
$$

for any complex coefficients $a, b, c, d$ such that $a d-b c \neq 0$. Then $f$ has only elliptic points.

Remark. We could use the integral $\int_{0}^{t} \log \left(1+\left|f\left(\pi_{s}\right)\right|^{2}\right) d s$ reminiscent of the proximity function associated to the value $\infty$.

Although the 3-dimensional geometric picture considered by Franchi (1999) is somewhat different, our study on automorphic functions requires a similar method. Moreover Lemma 4.2 below shows that we sum i.i.d. random variables whose law is almost the same as the one obtained in Franchi's lemma 2. Hence, we have only to rewrite parts of his work into a new 2-dimensional setting.

Lemma 4.2. Denote by $\sigma(\log (R-\varepsilon))$ the first hitting time of $\log (R-\varepsilon)$ by a Brownian motion $\left(b_{s}\right)$ with drift $-1 / 2$ started at $\log (R)$ and set $a=\sqrt{R}$ and $b=\sqrt{R-\varepsilon}$. The law of $Z:=\int_{0}^{\sigma(u)} \exp \left(b_{s}\right) d s$ is identical to the law of 
$4 \widehat{T}$ if $\widehat{T}:=\inf \left\{t \geq 0: R_{t}^{(-1)}=a\right\}$ where $\left(R_{t}^{(-1)}, t \leq \eta\right)$ is a 0-dimensional Bessel process with lifetime $\eta$, started at $b$. Moreover

$$
\mathbb{E}_{a}\left(\exp \left(-\frac{\lambda^{2}}{2} \widehat{T}\right)\right)=\frac{b K_{1}(b \lambda)}{a K_{1}(a \lambda)}
$$

if $K_{1}$ denotes the modified Bessel function with index 1.

Proof. Recall Lamperti's formula (Revuz \& Yor exercise XI.1.28): there exists a Bessel process $\left(R_{t}^{(\mu)}, t \leq \eta\right)$ with index $\mu$ started at 1 such that

$$
\exp \left(B_{t}+\mu t\right)=R^{(\mu)}\left(\int_{0}^{t} \exp \left(2 B_{s}+2 \mu s\right) d s\right) .
$$

Here $\eta$ denotes the finite lifetime if $\mu \leq-1$.

Hence, the first result is obtained by scaling. We may use the bounded martingale

$$
\left(\exp \left(-\frac{\lambda^{2}}{2} s\right) K_{1}\left(\lambda R_{s}^{(-1)}\right), s \leq \widehat{T}\right)
$$

to get the desired Laplace transform.

Remark. By using formula (5.7.11) in Lebedev we obtain the equivalent

$$
1-\mathbb{E}_{b}(\exp (-\lambda \widehat{T})) \sim \frac{\lambda}{2}\left(b^{2}-a^{2}\right) \log \left(\frac{1}{\lambda}\right)
$$

if $\lambda \longrightarrow 0^{+}$. In this particular case, the Karamata Tauberian Theorem (for instance result 8.1.7 in Bingham et al. monograph (1987)) gives only a weaker substitute

$$
\int_{0}^{x} t d F(t) \sim \frac{\left(b^{2}-a^{2}\right)}{2} \log (x), \quad \text { if } x \longrightarrow \infty .
$$

However the equivalent above is enough to deduce that $\widehat{T}$ belongs to the domain of attraction of a totally asymmetric Cauchy random variable.

Proof of Theorem 4.1. For the sake of notational simplicity, we first consider the case $f=h=\mu \circ I^{-1}$. We set $h:=\mu \circ I^{-1}$.

Fix $R>1 / 2,0<\varepsilon<R$ and set

$$
\rho(\varepsilon)=\lim _{t \rightarrow \infty} \frac{N_{t}}{t}
$$


where $N_{t}$ is the number of excursions performed before time $t$. With the same notations as in section 2.1, the outwards parts $\left(\Pi_{t}^{\prime}, S_{k} \leq t \leq\right.$ $\left.T_{k}\right)$ create i.i.d. random variable $\int_{S_{k}}^{T_{k}} \log ^{+}\left(\left|h\left(\pi_{s}\right)\right|\right) d s$. Denote by $Z_{k}:=$ $\int_{S_{k}}^{T_{k}} Y_{s} d s$. If $R$ is great enough,

$$
\left(\pi-\varepsilon_{1}\right) Z_{k} \leq \int_{S_{k}}^{T_{k}} \log ^{+}\left(\left|h\left(\pi_{s}\right)\right|\right) d s \leq\left(\pi+\varepsilon_{1}\right) Z_{k},
$$

hence we have to study the sums

$$
\sum_{k=1}^{n} \int_{S_{k}}^{T_{k}} Y_{s} d s
$$

Obviously the remaining part $\sum_{k=1}^{n} \int_{T_{k}}^{S_{k}} Y_{s} d s$ is $O(n)$. By lemma 4.2 and Franchi's lemma 2,

$$
\frac{1}{t \log (t)} \sum_{k=1}^{N_{t}} Z_{k} \stackrel{P}{\longrightarrow} 4 \frac{b^{2}-a^{2}}{2} \rho(\varepsilon)=2 \varepsilon \rho(\varepsilon) .
$$

Since the last incomplete excursion brings no trouble because we consider positive variables,

$$
\frac{1}{t \log (t)} \int_{0}^{t} \Im m\left(\Pi_{s}^{\prime}\right) d s \stackrel{P}{\longrightarrow} 2 \varepsilon \rho(\varepsilon) .
$$

Let now $\varepsilon$ decrease to 0 . By Franchi's lemma 3 where $\mu$ is replaced by the Haar measure

$$
\mu_{0}(d x d y)=\frac{d x d y}{2 \pi y^{2}}
$$

on $\mathcal{F}_{0}$, we get

$$
\lim _{\varepsilon \rightarrow 0} \mathbb{E}_{\log (R)}\left(\sigma(\log (R-\varepsilon)) \rho(\varepsilon)=\mu_{0}(\{y>R\})=\frac{1}{\pi R} .\right.
$$

But

$$
\mathbb{E}_{\log (R)}\left(\sigma(\log (R-\varepsilon))=2 \log \left(\frac{R}{R-\varepsilon}\right)\right.
$$

hence

$$
\lim _{\varepsilon \rightarrow 0} \varepsilon \rho(\varepsilon)=\frac{1}{2 \pi}
$$


If there are several parabolic points, we decompose the trajectory into the excursions between nested horoballs $H\left(p_{i}, R\right) \subset H\left(p_{i}, R-\varepsilon\right)$ with base point $p_{i}$. The trace of $\log ^{+}(|f|)$ on $\mathcal{F}-\cup_{i} H\left(p_{i}, R-\varepsilon\right) \cap \mathcal{F}$ defines an integrable additive functional with respect to the area measure. Indeed, the possible poles create logarithmic singularities.

We raise the following question:

Problem about fuchsians functions (and perhaps normal functions). Assume the existence of complex coefficients $a, b, c, d$ such that $a d-b c \neq 0$ and

$$
\frac{1}{t \log (t)} I\left(a f+\frac{b}{c f}+d, t\right) \stackrel{P}{\longrightarrow} \text { const }>0 \text {. }
$$

What can be deduced about

$$
\liminf _{r \rightarrow 1} \frac{T_{f}(r)}{\log \left(\frac{1}{1-r}\right)} ?
$$

Remark. If $f$ is Fuchsian of first kind and valence $n$, without any assumptions on the cusps,

$$
\frac{T_{f}(r)}{\log \left(\frac{1}{1-r}\right)}
$$

goes to a limit bounded by $6 n$. Indeed, it is known that $\operatorname{area}(\mathcal{F}) \geq \pi / 3$. Hence the study of the counterpart

$$
\limsup _{r \rightarrow 1} \frac{T_{f}(r)}{\log \left(\frac{1}{1-r}\right)}
$$

seems easier. This quantity is finite for a large class of functions, all normal functions for instance.

\section{Another example of a zero order function.}

Consider the function $\mu$ of Section 1.3 and define $\log (\mu)$ on its Riemann surface. This function misses a denumerable set of values. Nevanlinna (1929) showed that 


$$
\limsup _{r \rightarrow 1} \frac{T_{\log (\mu)}(r)}{\log \left(\log \left(\frac{1}{1-r}\right)\right)}<\infty
$$

and he conjectured that the liminf does not vanish.

Indeed, the stronger result holds:

\section{Theorem 5.3.}

$$
\lim _{r \rightarrow 1} \frac{T_{\log (\mu)}(r)}{\log \left(\log \left(\frac{1}{1-r}\right)\right)}=\text { const }>0 .
$$

Proof. Assume that $\mu(z)=\exp (a)$. Here $n_{\log (\mu)}(r, a)$ counts the number of $G_{0}$ orbits $g . z$ in $D_{r}$ such that the homology of $g$ with respect to zero is null. The word in the free group belongs to the set \{sum of the exponents associated to the letter $S=0\}$.

The problem of counting the number of $\Gamma_{2}$ orbits with a partial homological constraint was open in the noncompact case for a long time but Aaronson and Denker (1999) recently showed that

$$
\sum_{g \in G_{0}}(1-|g(0)|)^{s} \sim \text { Const } \log \left(\frac{1}{s-1}\right), \quad \text { as } s \longrightarrow 1+
$$

if our $G_{0}$ subgroup is denoted by $\Gamma(\mathbb{C} / \mathbb{Z})$. Hence

$$
\sum_{g \in G_{0}} \exp \left(-s d(0, g(0)) \sim \text { Const } \log \left(\frac{1}{s-1}\right) .\right.
$$

We obtain with the help of a Ikehara's type tauberian theorem that

$$
n(\log (\mu), r) \sim \frac{\text { const }}{(1-r) \log \left(\frac{1}{1-r}\right)}
$$

(or const $\exp (d) / d$ ), We use for instance the Ikehara \& Delange Tauberian theorem 12.4 of the appendix in Parry and Pollicott (1990). It follows easily that

$$
T_{\log (\mu)}(r) \sim \int_{1}^{r} n(\log (\mu), u) d u \sim \text { const } \log \left(\log \left(\frac{1}{1-r}\right)\right) .
$$


Acknoledgement. I am grateful to Yueyun $\mathrm{Hu}$ for helpful discussions.

\section{References.}

[1] Aaronson, J., Denker, M., The Poincaré series of $\mathbb{C} / \mathbb{Z}$. Ergod. Th. \& Dynam. Sys. 19 (1999), 1-20.

[2] Ahlfors, L., Complex Analysis. McGraw Hill, 1966.

[3] Atsuji, A., Nevanlinna theory via stochastic calculus. J. Funct. Anal. 132 (1995), 473-510.

[4] Atsuji, A., On the growth of meromorphic functions on the unit disk and conformal martingales. J. Math. Sci. Univ. Tokyo 3 (1996), 45-56.

[5] Bingham, N. H., Goldie, C. M., Teugels, J. L., Regular variation. Cambridge University Press, 1987.

[6] Carne, T. K., Brownian motion and Nevanlinna theory. Proc. London Math. Soc. 52 (1986), 349-68.

[7] ForD, L. R., Automorphic functions. Chelsea, 1951.

[8] Franchi, J., Asymptotic singular homology of a complete hyperbolic 3manifold of finite volume. Proc. London Math. Soc. 79 (1999), 451-480.

[9] Gruet, J.-C., On the length of the homotopic Brownian word in the thrice punctured sphere. Probab. Theor. Relat. Fields 111 (1998), 489-516.

[10] Itô, K., MCKean, H. P., Diffusion processes and their sample paths. Springer, 1974.

[11] LeBedev, N., Special functions and their applications. Dover, 1972.

[12] LeHner, J., Discontinuous groups and automorphic functions. American Mathematical Society, 1964.

[13] LyOns, T., A synthetic proof of Makarov's law of the iterated logarithm. Bull. London Math. Soc. 22 (1990), 159-162.

[14] Lyons, T. J., McKean, H. P., Winding of the plane Brownian motion. Adv. in Math. 51 (1984), 212-225.

[15] Melián, M. V., Pestana, D., Geodesic excursions into cusps in finite volume hyperbolic manifolds. Michigan Math. J. 40 (1993), 77-93.

[16] Nevanlinna, R., Le théorème de Picard-Borel et la théorie des fonctions méromorphes. Gauthier Villars, 1929.

[17] Nevanlinna, R., Analytic functions. Springer, 1970.

[18] Parry W., Pollicott, M., Zeta functions and the periodic orbit structure of hyperbolic dynamics. Astérisque 187-188, 1990.

[19] Patterson, S. J., A lattice point problem in hyperbolic space, Mathematica 22 (1975), 81-88.

[20] Pommerenke, Ch., On normal automorphic functions. Michigan J. Math. 21 (1974), 193-202. 
[21] Ratcliffe, J. G., Foundations of hyperbolic manifolds. Springer, 1994.

[22] Revuz, D., YoR, M., Continuous martingales and brownian motion. Springer, 1999.

[23] Schiff, J. L., Normal families. Universitext, Springer, 1993.

[24] Shea D. F., Sons, L. R., Value distribution theory for meromorphic functions of slow growth in the disk. Houston J. Math. 12 (1986), 249-266.

[25] Sons, L. R., Values for the normal meromorphic functions. J. London Math. Soc. 29 (1984), 89-93.

[26] Stratmann, B., Velani, S. L., The Patterson measure for geometrically finite groups with parabolic elements, new and old. Proc. London Math. Soc. 71 (1995), 197-220.

[27] Sullivan, D., Disjoint spheres, approximation by imaginary quadratic numbers and the logarithm law for geodesics. Acta Math. 149 (1982), 215-237.

[28] Terras, A., Harmonic analysis on symmetric spaces and applications. Vol. 1, Springer, 1985.

[29] TsujI, M., Potential theory in modern function theory. Maruzen, 1959.

Recibido: 1 de marzo de 2000

Jean-Claude Gruet

Laboratoire SDAD

B.P. 5186 Campus II

F-14032 Caen-cedex, FRANCE

gruet@math . unicaen.fr 